\title{
Advantages of NOMA for Multi-User BackCom Networks
}

\author{
Zhiguo Ding, Fellow, IEEE and H. Vincent Poor, Life Fellow, IEEE
}

\begin{abstract}
Ambient backscatter communication (BackCom) is faced with the challenge that a single BackCom device can occupy multiple orthogonal resource blocks unintentionally. As a result, in order to avoid co-channel interference, a conventional approach is to serve multiple BackCom devices in different time slots, which reduces both spectral efficiency and connectivity. This letter demonstrates that the use of non-orthogonal multiple access (NOMA) can efficiently improve the system throughput and support massive connectivity in ambient BackCom networks. In particular, two transceiver design approaches are developed in the letter to realize different tradeoffs between system performance and complexity.
\end{abstract}

Index Terms-Non-orthogonal multiple access, backscatter communications, space division multiple access, orthogonal frequency-division multiple access.

\section{INTRODUCTION}

Recently, various novel backscatter communication (BackCom) approaches have been developed to support the envisioned ultra-massive machine type communications (umMTC) [1]-[5]. The key idea of these BackCom approaches is to use the signals sent by users in a legacy system for exciting the circuits of BackCom devices. This type of BackCom is featured by the challenge that a single BackCom device can occupy multiple orthogonal resource blocks unintentionally. For example, if the legacy system is based on orthogonal frequency-division multiple access (OFDMA), a BackCom device needs to reflect all the legacy signals sent at different subcarriers, which means that a signal sent by the device appears at multiple subcarriers. A similar challenge also presents if space division multiple access (SDMA) is used in the legacy system. In order to avoid the co-channel interference between BackCom devices, orthogonal multiple access (OMA) approaches have been conventionally used, e.g., BackCom devices are served in different time slots [2], [3], which reduces both spectral efficiency and connectivity.

This letter is to demonstrate the advantage of using nonorthogonal multiple access (NOMA) to improve the system throughput and connectivity in BackCom networks. In particular, this letter considers a full-duplex (FD) network, where downlink users are viewed as legacy users and multiple uplink BackCom devices are served simultaneously in the downlink users' channels by using BackCom assisted NOMA (BACNOMA) [5]. Unlike [5], multiple legacy users are considered in this letter, where the use of both SDMA and OFDMA in the legacy system is investigated. Two transceiver design approaches are proposed in the letter, where one approach can realize the sum capacity of the multiple access channel (MAC) and the other one can be implemented in a low complexity manner. The provided simulation results demonstrate that

Z. Ding and H. V. Poor are with the Department of Electrical Engineering, Princeton University, Princeton, NJ 08544, USA. Z. Ding is also with the School of Electrical and Electronic Engineering, the University of Manchester, Manchester, UK (email: zhiguo.ding@manchester.ac.uk poor@princeton.edu.
BAC-NOMA realizes a larger throughput and support more devices than OMA, even if random resource allocation is used.

\section{SySTEM MODEL}

Consider an ambient BackCom network, where SDMA is used in the legacy system. In particular, in the legacy system, a base station serves $K$ downlink users, denoted by $\mathrm{U}_{k}$, via $K$ spatial beamforming vectors, denoted by $\mathbf{w}_{k}, 1 \leq k \leq K$. The application of BAC-NOMA can ensure that additional $M$ uplink BackCom devices, denoted by $\mathrm{BD}_{m}, 1 \leq m \leq$ $M$, are admitted to the bandwidth used by the legacy system [5]. It is assumed that each user/device is equipped with a single antenna, the base station has $N$ antennas with the fullduplex capability, and each uplink device is equipped with a BackCom circuit. We note that the proposed BAC-NOMA scheme can also been applied to the case with OFDMA based legacy systems, as shown in Section IV.

Denote $x_{k}$ by the signal sent by downlink user $\mathrm{U}_{k}$. Based on the principle of SDMA, the base station broadcasts the following superimposed signals: $\mathbf{s}_{0}=\sqrt{P_{0}} \sum_{k=1}^{K} \mathbf{w}_{k} x_{k}$, where $P_{0}$ denotes the transmit power of the base station [6]. Optimizing the transmission strategy of the legacy system, i.e., optimizing $\mathbf{w}_{k}$ and $P_{0}$, is beyond the scope of this letter, since it should happen before the uplink BackCom devices are admitted via BAC-NOMA.

Each downlink user receives the following:

$$
y_{k}=\mathbf{g}_{k}^{T} \mathbf{s}_{0}+\sum_{m=1}^{M} \sqrt{\eta_{m}} g_{m, k} \mathbf{h}_{m}^{T} \mathbf{s}_{0} s_{m}+n_{k},
$$

where $\mathbf{g}_{k}$ denotes the channel vector between the base station and downlink user $\mathrm{U}_{k}, \mathbf{h}_{m}$ denotes the channel vector between the base station and uplink device $\mathrm{BD}_{m}, g_{m, k}$ denotes the channel gain between $\mathrm{U}_{k}$ and $\mathrm{BD}_{m}, \eta_{m}$ denotes the BackCom reflection coefficient of $\mathrm{BD}_{m}$ [7], $s_{m}$ denotes the signal sent by $\mathrm{BD}_{m}$, and $n_{k}$ denotes the receiver noise. For notational convenience, it is assumed that the noise terms at different receivers have the same power, denoted by $\sigma^{2}$.

Because $s_{m}$ is unknown to the downlink users, the term, $I \triangleq \sum_{m=1}^{M} \sqrt{\eta_{m}} g_{m, k} \mathbf{h}_{m}^{T} \mathbf{s}_{0} s_{m}$, can be treated as interference, whose power is given by $\mathcal{E}_{x_{k}, s_{m}}\left\{I I^{*}\right\}=$ $P_{0} \sum_{m=1}^{M} \eta_{m}\left|g_{m, k}\right|^{2}\left|\mathbf{h}_{m}^{T} \mathbf{W}\right|^{2}$, where $\mathcal{E}\{\cdot\}$ denotes the expectation operation, and $\mathbf{W}=\left[\begin{array}{lll}\mathbf{w}_{1} & \cdots & \mathbf{w}_{K}\end{array}\right]$. Therefore, the data rate of downlink user $\mathrm{U}_{k}$ is given by

$$
R_{k}^{\mathrm{D}}=\log \left(1+\frac{P_{0}\left|\mathbf{g}_{k}^{T} \mathbf{w}_{k}\right|^{2}}{P_{0} \sum_{i=1, i \neq k}^{K}\left|\mathbf{g}_{k}^{T} \mathbf{w}_{i}\right|^{2}+\mathcal{E}_{x_{k}, s_{m}}\left\{I I^{*}\right\}+\sigma^{2}}\right) .
$$

The base station receives the following observation:

$$
\mathbf{y}_{\mathrm{BS}}=\sum_{m=1}^{M} \sqrt{\eta_{m}} \mathbf{h}_{m} \mathbf{h}_{m}^{T} \mathbf{s}_{0} s_{m}+\mathbf{s}_{\mathrm{SI}}+\mathbf{n}_{\mathrm{BS}}
$$

where $\mathbf{s}_{\mathrm{SI}}$ is assumed to be complex Gaussian distributed, i.e., $\mathbf{s}_{\mathrm{SI}} \sim C N\left(0, \alpha P_{0} \mathbf{C}_{\mathrm{SI}}\right), \mathbf{C}_{\mathrm{SI}}$ denotes the covariance matrix of 
the self-interference channels and $\alpha, 0 \leq \alpha \leq 1$, indicates the amount of the residual self-interference [8].

Depending on how successive interference cancellation (SIC) is carried out, different sum rates can be realized for the BackCom devices, as shown in the following section.

\section{Two Approaches With DifFERENT TRADEOFFS BETWEEN PERFORMANCE AND COMPLEXITY}

\section{A. Approach I - A Sum-Capacity Approaching Design}

By applying the noise pre-whitening process [9], i.e., applying a detection matrix $\left(\sigma^{2} \mathbf{I}_{N}+\alpha P_{0} \mathbf{C}_{\mathrm{SI}}\right)^{-\frac{1}{2}}$ to $\mathbf{y}_{\mathrm{BS}}$, the model in (2) can be rewritten as follows:

$$
\tilde{\mathbf{y}}_{\mathrm{BS}}=\sum_{m=1}^{M} \sqrt{\eta_{m}} \mathbf{h}_{m}^{T} \mathbf{s}_{0} \tilde{\mathbf{h}}_{m} s_{m}+\tilde{\mathbf{n}}_{\mathrm{BS}}=\tilde{\mathbf{H D}} \mathbf{s}+\tilde{\mathbf{n}}_{\mathrm{BS}}
$$

where $\tilde{\mathbf{y}}_{\mathrm{BS}}=\left(\sigma^{2} \mathbf{I}_{N}+\alpha P_{0} \mathbf{C}_{\mathrm{SI}}\right)^{-\frac{1}{2}} \mathbf{y}_{\mathrm{BS}}, \quad \tilde{\mathbf{h}}_{m}=$ $\left(\sigma^{2} \mathbf{I}_{N}+\alpha P_{0} \mathbf{C}_{\mathrm{SI}}\right)^{-\frac{1}{2}} \mathbf{h}_{m}, \quad \tilde{\mathbf{H}}=\left[\begin{array}{lll}\tilde{\mathbf{h}}_{1} & \cdots & \tilde{\mathbf{h}}_{M}\end{array}\right]$, $\tilde{\mathbf{n}}_{\mathrm{BS}}=\left(\sigma^{2} \mathbf{I}_{N}+\alpha P_{0} \mathbf{C}_{\mathrm{SI}}\right)^{-\frac{1}{2}}\left(\mathbf{s}_{\mathrm{SI}}+\mathbf{n}_{\mathrm{BS}}\right), \quad \mathbf{D}$ is a diagonal matrix with the elements on its main diagonal as $D_{m, m}=\sqrt{\eta_{m}} \mathbf{h}_{m}^{T} \mathbf{s}_{0}$, and $\mathbf{s}=\left[\begin{array}{lll}s_{1} & \cdots & s_{M}\end{array}\right]^{T}$.

By treating $\mathbf{D}$ as a power allocation matrix, the system model in (3) can be viewed as a special case of a conventional MAC, whose sum capacity can be realized as follows [9]. Without loss of generality, assume that $\mathrm{BD}_{m}$, signal is decoded at the $m$-th stage of SIC, where it is straightforward to show that different SIC decoding orders lead to the same sum capacity. Further assume that prior to decoding $\mathrm{BD}_{m}$ ' signal, the signals from $\mathrm{BD}_{i}, 1 \leq$ $i \leq m-1$, have been decoded correctly. By applying $\sqrt{\eta_{m}} \mathbf{h}_{m}^{H} \mathbf{s}_{0}^{*} \tilde{\mathbf{h}}_{m}^{H}\left(\mathbf{I}_{N}+\sum_{i=m+1}^{M} \eta_{i}\left|\mathbf{h}_{i}^{T} \mathbf{s}_{0}\right|^{2} \tilde{\mathbf{h}}_{i} \tilde{\mathbf{h}}_{i}^{H}\right)^{-1}$ as the detector, the following data rate is achievable to $\mathrm{BD}_{m}$ :

$$
\begin{aligned}
R_{m}^{\mathrm{MAC}}= & \log \operatorname{det}\left(\mathbf{I}_{N}+\left(\mathbf{I}_{N}+\sum_{i=m+1}^{M} \eta_{i}\left|\mathbf{h}_{i}^{T} \mathbf{s}_{0}\right|^{2} \tilde{\mathbf{h}}_{i} \tilde{\mathbf{h}}_{i}^{H}\right)^{-1}\right. \\
& \left.\times \eta_{m}\left|\mathbf{h}_{m}^{T} \mathbf{s}_{0}\right|^{2} \tilde{\mathbf{h}}_{m} \tilde{\mathbf{h}}_{m}^{H}\right)
\end{aligned}
$$

for $1 \leq m \leq M-1$, and $R_{M}^{\mathrm{MAC}}=$ $\log \operatorname{det}\left(\mathbf{I}_{N}+\eta_{M}\left|\mathbf{h}_{M}^{T} \mathbf{s}_{0}\right|^{2} \tilde{\mathbf{h}}_{M} \tilde{\mathbf{h}}_{m}^{H}\right)$. Therefore, the sum capacity of the uplink BackCom devices achieved by this type of SIC is given by

$$
R_{\mathrm{sum}}^{\mathrm{MAC}}=\log \operatorname{det}\left(\mathbf{I}_{N}+\sum_{m=1}^{M} \eta_{m}\left|\mathbf{h}_{m}^{T} \mathbf{s}_{0}\right|^{2} \tilde{\mathbf{h}}_{m} \tilde{\mathbf{h}}_{m}^{H}\right) .
$$

This letter considers a problem of throughput maximization for the BackCom devices, which can be formulated as follows:

$$
\begin{array}{ll}
\max _{\eta_{m}} & R_{\mathrm{sum}}^{\mathrm{MAC}} \\
\text { s.t. } & \sum_{m=1}^{M} \eta_{m}\left|g_{m, k}\right|^{2}\left|\mathbf{h}_{m}^{T} \mathbf{W}\right|^{2} \leq \tau_{k}, \quad 1 \leq k \leq K \\
& 0 \leq \eta_{m} \leq 1, \quad 1 \leq m \leq M,
\end{array}
$$

where $\tau_{k}$ indicates the tolerable interference experienced by downlink user $\mathrm{U}_{k}$. If $\mathrm{U}_{k}$ has a target data rate, denoted by $R_{k}$, one choice of $\tau_{k}$ is given by $\tau_{k}=$ $\frac{\left|\mathbf{g}_{k}^{T} \mathbf{w}_{k}\right|^{2}-\epsilon_{k} \sum_{i=1, i \neq k}^{K}\left|\mathbf{g}_{k}^{T} \mathbf{w}_{i}\right|^{2}}{\epsilon_{k}}-\frac{\sigma^{2}}{P_{0}}$, and $\epsilon_{k}=2^{R_{k}}-1$.

Remark 1: Problem P1 is a concave optimization problem since its objective function is in a log-det form and its constraints are affine [10]. Various optimization solvers, such as Matlab fmincon, can be straightforwardly applied to find the optimal solution of Problem P1.

Remark 2: The solution of Problem P1 is based on an instantaneous realization of $x_{k}$. Therefore, significant system overhead can be consumed for the base station to inform the BackCom devices about the optimal choices of $\eta_{m}^{*}$. A lowcomplexity alternative is to use random choices of $\eta_{m}$ which satisfy $(\mathrm{P} 1 \mathrm{~b})$ and $(\mathrm{P} 1 \mathrm{c})$. The simulation results provided in Section $\mathrm{V}$ show that Approach I with random $\eta_{m}$ can still significantly outperform OMA.

Remark 3: Another type of complexity introduced by Approach I is explained in the following. During each SIC step, $\sqrt{\eta_{m}} \mathbf{h}_{m}^{H} \mathbf{s}_{0}^{*} \tilde{\mathbf{h}}_{m}^{H}\left(\mathbf{I}_{N}+\sum_{i=m+1}^{M} \eta_{i}\left|\mathbf{h}_{i}^{T} \mathbf{s}_{0}\right|^{2} \tilde{\mathbf{h}}_{i} \tilde{\mathbf{h}}_{i}^{H}\right)^{-1}$ needs to be computed, where the inverse of the matrix requires a computational complexity of $\mathcal{O}\left(N^{3}\right)$. In the case of the number of antennas, $N$, is large, the computational complexity for generating this detector can be significant, which motivates the low-complexity approach introduced in the next section.

\section{B. Approach II - A Low-complexity QR Based Deign}

Assume that the composite channel matrix $\tilde{\mathbf{H}}$ in (3) can be decomposed via $\mathrm{QR}$ decomposition as follows: $\tilde{\mathbf{H}}=\mathbf{Q R}$, where $\mathbf{Q}$ is an $N \times N$ unitary matrix, and $\mathbf{R}$ is an $N \times M$ upper triangular matrix [11]. The base station can use $\mathbf{Q}^{H}$ as a detection matrix, which simplifies the system model in (3) as follows:

$$
\mathbf{Q}^{H} \tilde{\mathbf{y}}_{\mathrm{BS}}=\mathbf{R D} \mathbf{s}+\mathbf{Q} \tilde{\mathbf{n}}_{\mathrm{BS}} .
$$

By using the upper triangular structure of $\mathbf{R}$, SIC can be implemented in a low-complexity manner [11]. In particular, during the $(M-m+1)$-th step, the signal from $\mathrm{BD}_{m}$ can be decoded with the following data rate:

$$
R_{m}=\log \left(1+R_{m, m}^{2} \eta_{m}\left|\mathbf{h}_{m}^{T} \mathbf{s}_{0}\right|^{2}\right)
$$

where $R_{m, m}$ is defined similar to $D_{m, m}$. To further reduce system overhead, it is ideal to formulate the resource allocation problem based on the following average sum rate:

$$
\bar{R}_{\text {sum }}=\sum_{m=1}^{M} \mathcal{E}_{x_{k}}\left\{\log \left(1+R_{m, m}^{2} \eta_{m}\left|\mathbf{h}_{m}^{T} \mathbf{s}_{0}\right|^{2}\right)\right\} .
$$

Therefore, the considered long-term throughput maximization problem can be formulated as follows:

$$
\max _{\eta_{m}} \bar{R}_{\text {sum }} \text { s.t. } \mathrm{P} 1 \mathrm{~b}, \mathrm{P} 1 \mathrm{~b} \text {. }
$$

We note that there is an analogy between Problem P2 and the one developed for cognitive MAC [12]; however, the explicit expression of $\bar{R}_{\text {sum }}$ can be obtained, as shown in the following. First define $\bar{R}_{m}$ as follows:

$$
\bar{R}_{m}=\mathcal{E}_{x_{k}}\left\{\log \left(1+R_{m, m}^{2} \eta_{m} \mathbf{s}_{0}^{H} \mathbf{h}_{m}^{*} \mathbf{h}_{m}^{T} \mathbf{s}_{0}\right)\right\} .
$$


Recall that $\mathbf{s}_{0}=\sqrt{P_{0}} \sum_{k=1}^{K} \mathbf{w}_{k} x_{k}$, which means that $\bar{R}_{m}$ can be rewritten as follows:

$$
\bar{R}_{m}=\mathcal{E}_{x_{k}}\left\{\log \left(1+P_{0} R_{m, m}^{2} \eta_{m} \mathbf{x}^{H} \mathbf{W}^{H} \mathbf{h}_{m}^{*} \mathbf{h}_{m}^{T} \mathbf{W} \mathbf{x}\right)\right\} .
$$

A closed-form expression of $\bar{R}_{m}$ can be found by using the method developed in [13]:

$$
\begin{aligned}
\bar{R}_{m}= & \log (e) \mathcal{E}_{x_{k}}\left\{\int _ { 0 } ^ { \infty } \left(\frac{e^{-t}}{t}\right.\right. \\
& \left.\left.-\frac{1}{t} e^{-t\left(1+P_{0} R_{m, m}^{2} \eta_{m} \mathbf{x}^{H} \mathbf{W}^{H} \mathbf{h}_{m}^{*} \mathbf{h}_{m}^{T} \mathbf{W} \mathbf{x}\right)}\right) d t\right\},
\end{aligned}
$$

which can be simplified as follows:

$$
\begin{aligned}
\bar{R}_{m}= & \log (e) \int_{0}^{\infty}\left(\frac{e^{-t}}{t}\right. \\
& \left.-\frac{e^{-t}}{t} \mathcal{E}_{x_{k}}\left\{e^{-t P_{0} R_{m, m}^{2} \eta_{m} \mathbf{x}^{H} \mathbf{W}^{H} \mathbf{h}_{m}^{*} \mathbf{h}_{m}^{T} \mathbf{W} \mathbf{x}}\right\}\right) d t .
\end{aligned}
$$

Assuming that $x_{k}$ 's are independent and identically distributed (i.i.d.) complex Gaussian variables with mean zero and unit variance, $\bar{R}_{m}$ can be evaluated as follows:

$$
\begin{aligned}
\bar{R}_{m} & =\log (e) \int_{0}^{\infty}\left(\frac{e^{-t}}{t}-\frac{e^{-t}}{t\left(1+t P_{0} R_{m, m}^{2} \eta_{m}\left|\mathbf{h}_{m}^{T} \mathbf{W}\right|^{2}\right)}\right) d t \\
& =\log (e) f\left(P_{0} R_{m, m}^{2} \eta_{m}\left|\mathbf{h}_{m}^{T} \mathbf{W}\right|^{2}\right),
\end{aligned}
$$

where $f(x) \triangleq-e^{\frac{1}{x}} E_{i}\left(-\frac{1}{x}\right)$ and the last step follows from [14, 3.352.6] and $E_{i}(\cdot)$ denotes the exponential integral function. We note that an alternative way to obtain (13) is to treat $\mathbf{h}_{m}^{T} \mathbf{W} \mathbf{x}$ in 10 as a complex Gaussian variable with mean zero and variance $\left|\mathbf{h}_{m}^{T} \mathbf{W}\right|^{2}$.

So Problem $\mathrm{P} 2$ can be recast in the following equivalent form:

$$
\max _{\eta_{m}} \sum_{m=1}^{M}-e^{\frac{1}{P_{0} R_{m, m}^{2} \eta_{m}\left|\mathbf{h}_{m}^{T} \mathbf{W}\right|^{2}}} E_{i}\left(-\frac{1}{P_{0} R_{m, m}^{2} \eta_{m}\left|\mathbf{h}_{m}^{T} \mathbf{W}\right|^{2}}\right)
$$

s.t. $\mathrm{P} 1 \mathrm{~b}, \mathrm{P} 1 \mathrm{~b}$.

Although Problem $\mathrm{P3}$ contains the exponential integral function, it is still concave as shown in the following lemma.

Lemma 1. Problem $P 3$ is a concave optimization problem.

Proof. See Appendix A

Remark 4: Because Problem $\mathrm{P} 3$ is concave, it can be straightforwardly solved by using various optimization solvers. We note that the computation of the function $f(x)$ in 13 can be difficult even for a moderately small $x$. For example, for $x=0.0013, \frac{1}{x}=750$, and Matlab returns $f(750)=$ Inf. To overcome this computational issue, the following approximation of $f(x)$ is used for small $x$. Recall that $-E_{i}\left(-\frac{1}{x}\right)=\Gamma\left(0, \frac{1}{x}\right)$, where $\Gamma(\beta, y)$ denotes the incomplete gamma function and can be approximated as follows [14]:

$$
\Gamma(\beta, y) \approx y^{\beta-1} e^{-y} \sum_{m=0}^{L-1} \frac{(-1)^{m} \Gamma(1-\beta+m)}{y^{m} \Gamma(1-\beta)},
$$

for $y \rightarrow \infty$. By letting $L=1$ and $\beta=0, f(x)$ can be approximated as follows:

$$
f(x)=-e^{\frac{1}{x}} \Gamma\left(0, \frac{1}{x}\right) \approx x, \quad x \rightarrow 0,
$$

which can be used to approximate $(\mathrm{P} 3 \mathrm{a})$.

Remark 5: Compared to Approach I, the QR based design can be implemented with low computational complexity, as explained in the following. First, there is no need to calculate the inverse of a matrix with size of $N$ at each SIC step. Second, the resource allocation solution is not based on the instantaneous realizations of $x_{k}$, which reduces the system complexity. However, it is worth to point out that, unlike Approach I, Approach II cannot achieve the sum capacity of MAC, or support the overloading case, i.e., $M>N$.

\section{EXTENSION TO OFDMA-BASED LEGACY SystemS}

The aforementioned BAC-NOMA scheme can also be applied to the case, where OFDMA is used in the legacy system. In particular, in the considered legacy system, a base station serves $K$ downlink users, each denoted by $\mathrm{U}_{k}, 1 \leq k \leq K$, via $K$ orthogonal OFDMA subcarriers. If OMA is used, all the $K$ subcarriers will be occupied by a single BackCom device, since a signal reflected by one BackCom device can block all subcarriers. The application of BAC-NOMA can ensure that $M$ uplink BackCom devices are simultaneously admitted to share the subcarriers. For the purpose of illustration, it is assumed that each node is equipped with a single antenna.

Without loss of generality, assume that downlink user $\mathrm{U}_{k}$ is served at the $k$-th subcarrier. Following the ambient BackCom model in [2], [3], at subcarrier $k$, the frequency-domain baseband signal received by downlink user $\mathrm{U}_{k}$ is given by

$$
y_{k}^{\mathrm{D}}=\sqrt{P_{0}} G_{k} x_{k}+\sqrt{P_{0}} \sum_{m=1}^{M} \sqrt{\eta_{m}} G_{m, k} H_{m, k} x_{k} s_{m}+n_{k}^{\mathrm{D}},
$$

where $G_{k}$ denotes the channel gain between the base station and $\mathrm{U}_{k}$ at subcarrier $k, H_{m, k}$ denotes the forward channel gain from the base station to uplink device $\mathrm{BD}_{m}$ at subcarrier $k, G_{m, k}$ denotes the channel gain between $\mathrm{U}_{k}$ and $\mathrm{BD}_{m}$ at subcarrier $k$, and $n_{k}^{D}$ denotes the receiver noise.

Because $s_{m}$ is unknown to the downlink users, the term, $I_{o} \triangleq \sqrt{P_{0}} \sum_{m=1}^{M} \sqrt{\eta_{m}} G_{m, k}^{k} H_{m, k} x_{k} s_{m}$, is again treated as interference. The power of this interference term is given by $\mathcal{E}_{x_{k}, s_{m}}\left\{I_{o} I_{o}^{*}\right\}=P_{0} \sum_{m=1}^{M} \eta_{m}\left|G_{m, k}\right|^{2}\left|H_{m, k}\right|^{2}$. Therefore, the data rate of downlink user $\mathrm{U}_{k}$ is given by

$$
R_{k}^{\mathrm{D}}=\log \left(1+\frac{P_{0}\left|G_{k}\right|^{2}}{P_{0} \sum_{m=1}^{M} \eta_{m}\left|G_{m, k}\right|^{2}\left|H_{m, k}\right|^{2}+\sigma^{2}}\right) .
$$

At the base station, the frequency-domain baseband signal at the $k$-th subcarrier is given by

$$
y_{k}^{\mathrm{BS}}=\sqrt{P_{0}} \sum_{m=1}^{M} \sqrt{\eta_{m}} F_{m, k} H_{m, k} x_{k} s_{m}+s_{k}^{\mathrm{SI}}+n_{k}^{\mathrm{BS}},
$$

where $F_{m, k}$ denotes $\mathrm{BD}_{m}$ 's backward channel gain at subcarrier $k, s_{k}^{\mathrm{SI}}$ denotes the self-interference and $n_{k}^{\mathrm{BS}}$ denote the noise. As in the previous section, it is assumed that $s_{k}^{\mathrm{SI}} \sim$ 
$C N\left(0, \alpha P_{0}\left|h_{\mathrm{SI}}^{k}\right|^{2}\right)$, where $h_{\mathrm{SI}}^{k}$ denotes the self-interference channel. Furthermore, it is assumed that self-interference at different subcarriers is independent.

By applying the pre-whitening process, the system model at the base station can be expressed as follows:

$$
\breve{y}_{k}^{\mathrm{BS}}=\sqrt{P_{0}} x_{k} \breve{\mathbf{h}}_{k}^{H} \boldsymbol{\eta}^{\frac{1}{2}} \mathbf{s}+\breve{n}_{k}^{\mathrm{BS}},
$$

where $\breve{y}_{k}^{\mathrm{BS}}=\left(\alpha P_{0}\left|h_{\mathrm{SI}}^{k}\right|^{2}+\sigma^{2}\right)^{-\frac{1}{2}} y_{k}^{\mathrm{BS}}, \quad \breve{\mathbf{h}}_{k}=$ $\left(\alpha P_{0}\left|h_{\mathrm{SI}}^{k}\right|^{2}+\sigma^{2}\right)^{-\frac{1}{2}}\left[\begin{array}{lll}F_{1, k} H_{1, k} & \cdots & F_{M, k} H_{M, k}\end{array}\right]^{H}$, $\mathbf{s}=\left[\begin{array}{lll}s_{1} & \cdots & s_{M}\end{array}\right]^{T}, \boldsymbol{\eta}$ is an $M \times M$ diagonal matrix, i.e., $\boldsymbol{\eta}=\operatorname{diag}\left\{\eta_{1}, \cdots, \eta_{M}\right\}$, and $\breve{n}_{k}^{\mathrm{BS}}$ is a complex Gaussian white noise with mean zero and unit variance. Stacking the $K$ observations in one vector, the system model at the base station can be rewritten as follows:

$$
\breve{\mathbf{y}}^{\mathrm{BS}}=\sqrt{P_{0}} \mathbf{D}_{x} \breve{\mathbf{H}}^{H} \boldsymbol{\eta}^{\frac{1}{2}} \mathbf{S}+\breve{\mathbf{n}}^{\mathrm{BS}},
$$

where $\mathbf{D}_{x}$ is an $K \times K$ diagonal matrix, i.e., $\mathbf{D}_{x}=$ $\operatorname{diag}\left\{x_{1}, \cdots, x_{K}\right\}, \quad \breve{\mathbf{H}}=\left[\begin{array}{lll}\breve{\mathbf{h}}_{1} & \cdots & \breve{\mathbf{h}}_{K}\end{array}\right], \breve{\mathbf{y}}^{\mathrm{BS}}=$ $\left[\begin{array}{lll}\breve{y}_{1}^{\mathrm{BS}} & \cdots & \breve{y}_{K}^{\mathrm{BS}}\end{array}\right]^{T}$, and $\breve{\mathbf{n}}^{\mathrm{BS}}$ is constructed similarly to $\breve{\mathbf{y}}^{\mathrm{BS}}$.

By defining $\overline{\mathbf{H}}=\sqrt{P_{0}} \mathbf{D}_{x} \breve{\mathbf{H}}^{H}$ and treating $\boldsymbol{\eta}^{\frac{1}{2}}$ as a power allocation matrix, one can view the system model in (19) as a special case of conventional MAC, whose sum capacity can be realized as follows. Without loss of generality, assume that $\mathrm{BD}_{m}$ ' signal is decoded at the $m$-th stage of SIC, where it is noted that that different SIC decoding orders lead to the same sum capacity. Further assume that prior to decoding $\mathrm{BD}_{m}$, signal, the signals from $\mathrm{BD}_{i}, 1 \leq i \leq m-1$, have been decoded correctly. By applying $\overline{\mathbf{h}}_{m}^{H}\left(\mathbf{I}_{K}+\sum_{i=m+1}^{M} \eta_{i} \overline{\mathbf{h}}_{i} \overline{\mathbf{h}}_{i}^{H}\right)^{-1}$ as the detector, the following data rate is achievable to $\mathrm{BD}_{m}$ :

$$
\begin{aligned}
& R_{m}^{\mathrm{MAC}}= \\
& \log \operatorname{det}\left(\mathbf{I}_{K}+\left(\mathbf{I}_{K}+\sum_{i=m+1}^{M} \eta_{i} \overline{\mathbf{h}}_{i} \overline{\mathbf{h}}_{i}^{H}\right)^{-1} \eta_{m} \overline{\mathbf{h}}_{m} \overline{\mathbf{h}}_{m}^{H}\right),
\end{aligned}
$$

for $1 \leq m \leq M-1$, and $R_{M}^{\mathrm{MAC}}=\log \operatorname{det}\left(\mathbf{I}_{K}+\eta_{M} \overline{\mathbf{h}}_{M} \overline{\mathbf{h}}_{m}^{H}\right)$, where $\overline{\mathbf{h}}_{i}$ denotes the $i$-th column of $\overline{\mathbf{H}}$. Therefore, the sum capacity of the uplink BackCom devices is given by

$$
R_{\mathrm{sum}}^{\mathrm{MAC}}=\log \operatorname{det}\left(\mathbf{I}_{K}+\sum_{m=1}^{M} \eta_{m} \overline{\mathbf{h}}_{m} \overline{\mathbf{h}}_{m}^{H}\right),
$$

which can be used to formulate the following throughput maximization problem:

$$
\begin{aligned}
\max _{\eta_{m}} & R_{\text {sum }}^{\mathrm{MAC}} \\
\text { s.t. } & \sum_{m=1}^{M} \eta_{m}\left|G_{m, k}\right|^{2}\left|H_{m, k}\right|^{2} \leq \tau_{k}, \quad 1 \leq k \leq K \\
& \mathbb{\text { P1c } .} .
\end{aligned}
$$

Similar to $(\mathrm{P} 1 \mathrm{a}, \mathrm{P4a}$ is also in the concave log-det form. Therefore, Problem P4 is also concave and hence can be straightforwardly solved.

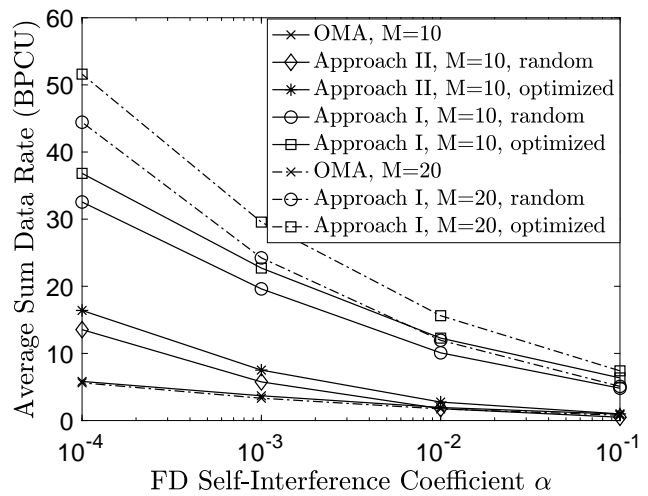

Fig. 1. The impact of FD self-interference on the performance of the considered transmission schemes. BPCU denotes bits per channel use. Case $\mathrm{I}$ is used for the locations of the downlink users and $K=N=10$.

\section{Simulation Results}

In this section, simulation results are presented to evaluate the performance of the proposed BAC-NOMA schemes. For the presented results, the path loss exponent is set as $3, P_{0}=$ $20 \mathrm{dBm}, \tau_{k}=0.01, \sigma_{n}^{2}=-94 \mathrm{dBm}$, and the base station is located at $(0,0) \mathrm{m}$. The uplink BackCom devices are randomly located inside a square of side $6 \mathrm{~m}$ whose center is located at $(0,0) \mathrm{m}$. Two cases, termed Cases I and II, are considered for the locations of the downlink users. For Case I, the downlink users are randomly located inside a square of side $6 \mathrm{~m}$ with its center located at $(0,0) \mathrm{m}$, whereas for case II, the users are also in the same size square with its center at $(3,0) \mathrm{m}$.

In Fig. 1 the impact of FD self-interference on the performance of the considered transmission schemes is studied. As can be seen from the figure, the NOMA schemes can outperform the OMA scheme, since multiple uplink BackCom devices can be simultaneously supported by the NOMA schemes. Furthermore, the figure shows that Approach I outperforms Approach II and also is applicable to the overloading cases, i.e., $M>N$. However, it is worth to point out that the performance gain of Approach I is obtained at a price of more system complexity, as discussed in Remarks 2 and 3. In addition, Fig. 1 1 shows that increasing $\alpha$ decreases the performance of all schemes, which is due to the fact that a larger $\alpha$ means more residual FD self-interference and hence leads to more performance degradation.

In Fig. 2 the impact of the number of BackCom devices on the performance of the considered schemes is studied. With more BackCom devices participating in NOMA transmission, the performance of the BAC-NOMA schemes is improved, whereas the impact of $M$ on the performance of OMA is insignificant. Another important observation from Fig. 2 is that the performance of the schemes for Case II is better than that of Case I, which is due to the fact that the interference between the uplink and downlink users is more severe in Case I. As discussed in Section IV] the concept of BACNOMA can be straightforwardly extended to the case with OFDMA based legacy systems, which is demonstrated in Fig. 3 Because $K$ subcarriers are used in the considered legacy system, the normalized sum-rate, i.e., $\frac{R_{\mathrm{sum}}^{\mathrm{MAC}}}{K}$, is used as the metric for the performance evaluation. As can be observed from Fig. 3, the BAC-NOMA schemes can outperform OMA, and inviting more BackCom devices to participate in NOMA 


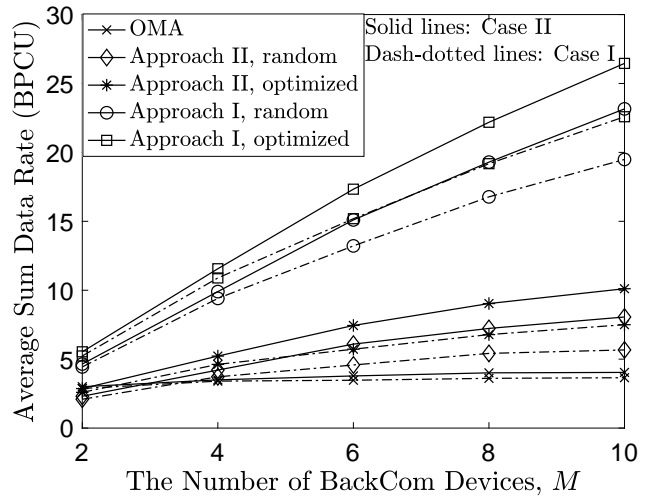

Fig. 2. The impact of the number of BackCom devices, $M$, on the performance of the considered transmission schemes. $\alpha=0.001$.

transmission improves the throughput, which are consistent to the observations made in Figs. 1 and 2

\section{CONClusions}

This letter has demonstrated the advantages of NOMA for ambient BackCom networks with OFDMA and SDMA used in legacy systems. By using BAC-NOMA, multiple BackCom devices can be served simultaneously, instead of being served in different time slots as in OMA. Two resource allocation approaches have been proposed in order to realize different tradeoffs between system performance and complexity.

\section{APPENDIX A}

\section{PROOF FOR LEMMA 1}

Recall that all the constraints of Problem P3 are affine, and therefore the lemma can be proved by showing that the objective function of Problem $\mathrm{P3}$ is concave. Recall that $f(x)=-e^{\frac{1}{x}} E_{i}\left(-\frac{1}{x}\right), x \geq 0$. The concavity of the objective function in $\mathrm{P3}$ a can be proved by showing that $f(x)$ is a concave function. Recall that the first order derivative of $f(x)$ has been obtained in [5] as follows:

$$
f^{\prime}(x)=e^{x^{-1}} x^{-2} E_{i}\left(-x^{-1}\right)+x^{-1} .
$$

By using (21) and with some algebraic manipulations, the second order derivative $f^{\prime \prime}(x)$ can be expressed as follows:

$$
\begin{aligned}
& f^{\prime \prime}(x)=e^{x^{-1}} x^{-4} \\
& \times\left(-E_{i}\left(-x^{-1}\right)-x e^{-x^{-1}}-2 x E_{i}\left(-x^{-1}\right)-x^{2} e^{-x^{-1}}\right) .
\end{aligned}
$$

Define $u(x)=-E_{i}\left(-x^{-1}\right)-x e^{-x^{-1}}-2 x E_{i}\left(-x^{-1}\right)-$ $x^{2} e^{-x^{-1}}$. In order to show $f^{\prime \prime}(x) \leq 0$, it is sufficient to show that $u(x) \leq 0$, since $x \geq 0$. The first order derivative of $u(x)$ is given by

$$
\begin{aligned}
u^{\prime}(x)= & e^{-x^{-1}} x^{-1}-e^{-x^{-1}}-e^{-x^{-1}} x^{-1}-2 E_{i}\left(-x^{-1}\right) \\
& +2 x e^{-x^{-1}} x^{-1}-2 x e^{-x^{-1}}-e^{-x^{-1}} \\
= & -2 E_{i}\left(-x^{-1}\right)-2 x e^{-x^{-1}},
\end{aligned}
$$

where the first step follows from the fact that $\frac{d E_{i}\left(-x^{-1}\right)}{d x}=$ $-\frac{e^{-x^{-1}}}{x}$.

Define $g(x) \triangleq E_{i}\left(-x^{-1}\right)+x e^{-x^{-1}}$, and $u^{\prime}(x)$ can be shown as a function of $g(x)$ as follows:

$$
u^{\prime}(x)=-2\left(E_{i}\left(-x^{-1}\right)+x e^{-x^{-1}}\right)=-2 g(x) .
$$

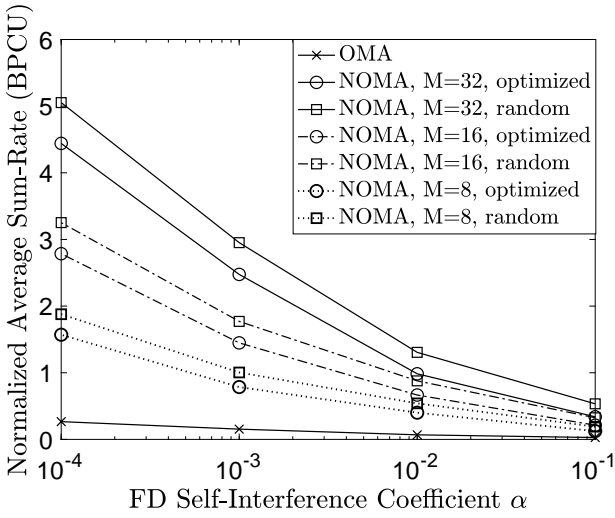

Fig. 3. The performance of BAC-NOMA in OFDMA based legacy systems. Case I is used for the locations of the downlink users. $N=K=16$.

In [5], it is shown that $g(x) \geq 0$ for $x \geq 0$. Therefore, $u^{\prime}(x) \leq$ 0 for $x \geq 0$, i.e., $u(x)$ is a monotonically decreasing function of $x$, which leads to the following inequality:

$$
\begin{aligned}
u(x) & =-E_{i}\left(-x^{-1}\right)-x e^{-x^{-1}}-2 x E_{i}\left(-x^{-1}\right)-x^{2} e^{-x^{-1}} \\
& \leq u(0)=0 .
\end{aligned}
$$

Because $u(x)$ is non-positive, the second order derivative of $f(x)$ is also non-positive. Therefore, $f(x)$ is concave and hence the objective function of Problem $\mathrm{P3}$ is also concave since a non-negative weighted sum of concave functions is still concave. The proof for the lemma is complete.

\section{REFERENCES}

[1] X. You, C. Wang, J. Huang et al., "Towards 6G wireless communication networks: Vision, enabling technologies, and new paradigm shifts," Sci. China Inf. Sci., vol. 64, no. 110301, pp. 1-74, Feb. 2021.

[2] G. Yang, D. Yuan, Y.-C. Liang, R. Zhang, and V. C. M. Leung, "Optimal resource allocation in full-duplex ambient backscatter communication networks for wireless-powered IoT," IEEE Internet of Things Journal, vol. 6, no. 2, pp. 2612-2625, Apr. 2019.

[3] R. Duan, R. Jantti, M. ElMossallamy, Z. Han, and M. Pan, "Multiantenna receiver for ambient backscatter communication systems," in Proc. IEEE International Workshop on Signal Processing Advances in Wireless Communications (SPAWC), Kalamata, Greece, 2018.

[4] R. Long, Y. Liang, H. Guo, G. Yang, and R. Zhang, "Symbiotic radio: A new communication paradigm for passive internet of things," IEEE Internet of Things Journal, vol. 7, no. 2, pp. 1350-1363, 2020.

[5] Z. Ding and H. V. Poor, "On the application of BAC-NOMA to 6G umMTC," IEEE Commun. Lett., to appear in 2021, Available on-line at arXiv:2102.06584, 2021.

[6] M. Vaezi, Z. Ding, and H. V. Poor, Multiple Access Techniques for $5 G$ Wireless Networks and Beyond. Springer International Publishing, 2019.

[7] F. D. Ardakani and V. W. Wong, "Joint reflection coefficient selection and subcarrier allocation for backscatter systems with NOMA," in Proc. IEEE Wireless Commun. and Networking Conf. (WCNC), Seoul, South Korea, 2020.

[8] D. W. K. Ng, Y. Wu, and R. Schober, "Power efficient resource allocation for full-duplex radio distributed antenna networks," IEEE Trans. Wireless Commun., vol. 15, no. 4, pp. 2896-2911, Apr. 2016.

[9] A. Goldsmith, S. A. Jafar, N. Jindal, and S. Vishwanath, "Capacity limits of MIMO channels," IEEE J. Select. Areas Commun., vol. 21, pp. 684702, Jun. 2003

[10] L. Vandenberghe, S. Boyd, and S.-P. Wu, "Determinant maximization with linear matrix inequality constraints," SIAM Journal on Matrix Analysis and Applications, vol. 19, no. 2, pp. 499-533, 1998.

[11] D. Wubben, R. Bohnke, V. Kuhn, and K.-D. Kammeyer, "MMSE extension of V-BLAST based on sorted QR decomposition," in Proc. IEEE Veh. Tech. Conf., Orlando, FL, USA, 2003.

[12] R. Zhang, S. Cui, and Y.-C. Liang, "On ergodic sum capacity of fading cognitive multiple-access and broadcast channels," IEEE Trans. Inform. Theory, vol. 55, no. 11, pp. 5161-5178, Nov. 2009.

[13] D. A. Basnayaka, P. J. Smith, and P. A. Martin, "Ergodic sum capacity of macrodiversity MIMO systems in flat rayleigh fading," IEEE Trans. Inform. Theory, vol. 59, no. 9, pp. 5257-5270, Sept. 2013.

[14] I. S. Gradshteyn and I. M. Ryzhik, Table of Integrals, Series and Products, 6th ed. New York: Academic Press, 2000. 